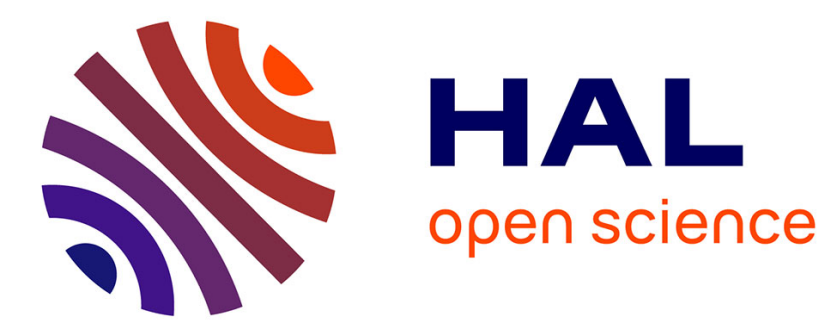

\title{
Smoothing PLLs for QAM Dynamical Phase Estimation
} Jianxiao Yang, Benoit Geller, Cedric Herzet, Jean-Marc Brossier

\section{To cite this version:}

Jianxiao Yang, Benoit Geller, Cedric Herzet, Jean-Marc Brossier. Smoothing PLLs for QAM Dynamical Phase Estimation. ICC 2009 - IEEE International Conference on Communications, Jun 2009, Dresden, Germany. pp.100, 10.1109/ICC.2009.5199465 . hal-01224236

\section{HAL Id: hal-01224236 \\ https://hal-ensta-paris.archives-ouvertes.fr/hal-01224236}

Submitted on 4 Dec 2015

HAL is a multi-disciplinary open access archive for the deposit and dissemination of scientific research documents, whether they are published or not. The documents may come from teaching and research institutions in France or abroad, or from public or private research centers.
L'archive ouverte pluridisciplinaire HAL, est destinée au dépôt et à la diffusion de documents scientifiques de niveau recherche, publiés ou non, émanant des établissements d'enseignement et de recherche français ou étrangers, des laboratoires publics ou privés. 


\title{
Smoothing PLLs for QAM Dynamical Phase Estimation
}

\author{
J. Yang, B. Geller, C. Herzet, and J.M. Brossier ${ }^{1}$
}

\begin{abstract}
This paper presents a near-optimum, low-complexity, fixed-interval smoothing algorithm that approaches the performance of an optimal smoother for the price of two lowcomplexity sequential estimators (two PLLs). The proposed Smoothing PLL (S-PLL) algorithm is easy to implement and fits the Cramer-Rao bounds over a wide range of signal-to-noise ratios. Moreover we show that, compared to the conventional forward loop, the proposed scheme allows to have a large gain of several dBs and is able to track frequency offsets.
\end{abstract}

Keywords-Dynamical Phase Estimation; Phase-Locked Loop (PLL); QAM; Smoothing Algorithm.

\section{INTRODUCTION}

Due to the increasing requirements of modern communication systems to face the physical channel (low signal-to-noise ratio, high data rates), phase estimation is more challenging than ever before. Since phase errors rapidly degrade the overall performance of communication systems, synchronization has recently become one of the most challenging tasks that a digital receiver has to cope with.

Noels et al [1],[2]. derived a maximum likelihood (ML) algorithm for the problem of constant phase estimation, and then applied a first-order and a second order phase-locked loop (PLL) based algorithm for the coded BPSK and QPSK dynamical phase estimation. The corresponding performances are limited both by the on-line bound and by a non-zero phase MSE floor. On the contrary, this paper deals with the non data aided (NDA) estimation of a time-varying phase and proposes an off-line Smoothing PLL (S-PLL) algorithm. To assess the performance of such algorithms, Bayesian and hybrid CramérRao Bounds (BCRB and HCRB) associated to this dynamical phase synchronization problem have already been considered in some recent contributions [3]-[6] and clearly show the superiority of the off-line scenario [7].

In practice, on-line estimators are often considered for complexity considerations. Among the famous algorithms, phase-locked loops are recognized low-cost devices for on-line estimation and have been integrated in many existing systems [8]-[11]. Despite a poor transient behaviour, their excellent asymptotic (i.e. tracking) characteristic makes them a reference from the performance-complexity trade-off point of view. The

\footnotetext{
${ }^{1}$ This work was partially funded by the ANR LURGA program.

J. Yang is with SATIE, ENS Cachan, (e-mail: shawn.yang@satie.ens-cachan.fr)

B. Geller is with LEI, ENSTA ParisTech (e-mail: geller@ensta.fr)

C. Herzet is with IRISA, INRIA Rennes (e-mail: cedric.herzet@irisa.fr)

J.M. Brossier is with GIPSA, Grenoble INP, (e-mail: jean-marc.brossier@gipsa-lab.inpg.fr)
}

poor transient behaviour has now been handled by several authors for many years (see e.g. [12].). The good performance of such PLLs can even be improved at low SNRs within the turbo-receiver framework (see e.g. [1],.[2],,[13].) but this paper is definitely not concerned about the code-aided framework. [14] proposed a CA belief-propagation (BP) algorithm for the BPSK dynamical phase estimation but the computation complexity of the proposed BP algorithm is rather high. This paper is concerned with a very simple synchronizing scheme for any QAM modulated signal which is able to operate near the off-line time-varying phase bounds. To our knowledge, it was first proposed without any justification and without any performance evaluation in [15]-[17]; contrarily to [1].,[2], it takes advantage of averaging two phase trajectories provided by two PLLs, so that this S-PLL algorithm is able to have such a near off-line Cramér-Rao bound performance.

The rest of the paper is organized as follows. In section II, we give the system model. In section III, we derive from the MAP estimation theory the proposed algorithm where the smoothing effect is achieved through two PLLs working in opposite time directions. Finally in section IV, we present the simulation results before giving some conclusions.

\section{SYSTEM MODEL}

We consider the transmission of a complex-valued QAM modulated sequence $\mathbf{c}=\left[c_{1}, \cdots, c_{\mathrm{K}}\right]^{T} \quad\left(c_{k} \in \mathbf{S}_{\mathrm{M}}\right)$ over an AWGN channel affected by some carrier phase offsets stacked in a vector $\boldsymbol{\theta}=\left[\theta_{1}, \cdots, \theta_{\mathrm{K}}\right]^{T}$. Assuming that the timing recovery is perfect without any inter-symbol interference (ISI), the sampled baseband signal $\mathbf{y}=\left[y_{1}, \cdots, y_{\mathrm{K}}\right]^{T}$ is written as:

$$
y_{k}=c_{k} e^{j \theta_{k}}+n_{k},
$$

where $c_{k}, \theta_{k}$ and $n_{k}$ are respectively the i.i.d. $k$-th unknown transmitted constellation symbol $\left(p\left(c_{k}\right)=1 / \mathrm{M}\right)$, the residual phase distortion and the zero mean complex-valued circular Gaussian noise with known variance $\sigma_{n}^{2}$. We suppose that the system operates in a non-data aided (NDA) mode. Hence, the conditional probability based on the known phase $\theta_{k}$ is:

$$
\begin{aligned}
& p\left(y_{k} \mid \theta_{k}\right)=\sum_{c_{k}} p\left(y_{k} \mid \theta_{k}, c_{k}\right) p\left(c_{k}\right)=\frac{1}{\mathrm{M} \pi \sigma_{n}^{2}} \exp \left\{-\frac{\left|y_{k}\right|^{2}}{\sigma_{n}^{2}}\right\} \sum_{c_{k} \in \mathbf{S}_{\mathrm{M}}} \Omega\left(c_{k}, y_{k}, \theta_{k}\right), \\
& \text { where } \Omega\left(c_{k}, y_{k}, \theta_{k}\right)=\exp \left\{\frac{-\left|c_{k}\right|^{2}+2 \operatorname{Re}\left\{y_{k} c_{k}^{*} e^{-j \theta_{k}}\right\}}{\sigma_{n}^{2}}\right\} .
\end{aligned}
$$

In practice, due to the rapid variant channel and the imperfections of the functional blocks before the phase 
estimation, the residual phase distortion can be modeled efficiently by a Brownian motion. The corresponding phase model is:

$$
\theta_{k}=\theta_{k-1}+\xi+w_{k},
$$

where $\theta_{k}$ is the unknown phase offset at time $k, \xi$ is the unknown constant frequency offset (linear drift), $w_{k}$ is a realvalued white Gaussian noise with zero mean and variance $\sigma_{w}^{2}$. This model is commonly used [18]-[21] in order to describe the behavior of practical oscillators for which the frequency is randomly perturbed. Based on (3), the corresponding conditional probability can be expressed as:

$$
p\left(\theta_{k} \mid \theta_{k-1}, \xi\right)=\frac{1}{\sqrt{2 \pi} \sigma_{w}} \exp \left\{-\frac{\left(\theta_{k}-\theta_{k-1}-\xi\right)^{2}}{2 \sigma_{w}^{2}}\right\} .
$$

\section{RATIONALE FOR A FORWARD / BACKWARD APPROACH BY MAP ESTIMATION THEORY}

In the MAP estimation approach, one classically chooses $\hat{\boldsymbol{\theta}}$ to maximize the posterior pdf [22]:

$$
\hat{\boldsymbol{\theta}}=\arg \max _{\boldsymbol{\theta}} p(\boldsymbol{\theta} \mid \mathbf{y})=\arg \max _{\boldsymbol{\theta}}[\ln p(\mathbf{y} \mid \boldsymbol{\theta})+\ln p(\boldsymbol{\theta})]
$$

Based on the model described by (2) and (4), the joint pdf of the observations and the parameters can be written as:

$$
\begin{aligned}
p(\mathbf{y}, \boldsymbol{\theta}, \boldsymbol{\xi})= & p(\mathbf{y} \mid \boldsymbol{\theta}) p(\boldsymbol{\theta} \mid \xi) \\
= & p\left(\theta_{1}\right) p\left(y_{1} \mid \theta_{1}\right) \prod_{k=2}^{\mathrm{K}} p\left(y_{k} \mid \theta_{k}\right) p\left(\theta_{k} \mid \theta_{k-1}, \xi\right) \\
= & \left(\frac{1}{M \pi \sigma_{n}^{2}}\right)^{\mathrm{K}}\left(\frac{1}{\sqrt{2 \pi} \sigma_{w}}\right)^{\mathrm{K}-1} p\left(\theta_{1}\right) \exp \left\{-\frac{\left|y_{1}\right|^{2}}{\sigma_{n}^{2}}\right\}\left(\sum_{c_{1} \in \mathbf{S}_{\mathrm{M}}} \Omega\left(c_{1}, y_{1}, \theta_{1}\right)\right) \\
& \cdot \prod_{k=2}^{\mathrm{K}} \exp \left\{-\frac{\left|y_{k}\right|^{2}}{\sigma_{n}^{2}}-\frac{\left(\theta_{k}-\theta_{k-1}-\xi\right)^{2}}{2 \sigma_{w}^{2}}\right\}\left(\sum_{c_{k} \in \mathbf{S}_{\mathrm{M}}} \Omega\left(c_{k}, y_{k}, \theta_{k}\right)\right)
\end{aligned}
$$

It is easy to obtain for any $\theta_{k}$ the first derivative of $\ln [p(\mathbf{y}, \boldsymbol{\theta}, \xi)]:$

$$
\begin{gathered}
\frac{\partial \ln [p(\mathbf{y}, \boldsymbol{\theta}, \xi)]}{\partial \theta_{1}}=\frac{\partial \ln \left[p\left(\theta_{1}\right)\right]}{\partial \theta_{1}}+\frac{\sum_{c_{1} \in \mathbf{S}_{\mathrm{M}}} \frac{\partial \Omega\left(c_{1}, y_{1}, \theta_{1}\right)}{\partial \theta_{1}}}{\sum_{c_{1} \in \mathbf{S}_{\mathrm{M}}} \Omega\left(c_{1}, y_{1}, \theta_{1}\right)}+\frac{\left(\theta_{2}-\theta_{1}-\xi\right)}{\sigma_{w}^{2}}, \\
\frac{\partial \ln [p(\mathbf{y}, \boldsymbol{\theta}, \xi)]}{\partial \theta_{k}}=\frac{\sum_{c_{k} \in \mathbf{S}_{\mathrm{M}}} \frac{\partial \Omega\left(c_{k}, y_{k}, \theta_{k}\right)}{\partial \theta_{k}}}{\sum_{c_{k} \in \mathbf{S}_{\mathrm{M}}} \Omega\left(c_{k}, y_{k}, \theta_{k}\right)}-\frac{\left(2 \theta_{k}-\theta_{k-1}-\theta_{k+1}\right)}{\sigma_{w}^{2}} \\
\frac{\partial \ln [p(\mathbf{y}, \boldsymbol{\theta}, \xi)]}{\partial \theta_{\mathrm{K}}}=\frac{\sum_{c_{\mathrm{K}} \in \mathbf{S}_{\mathrm{M}}} \frac{\partial \Omega\left(c_{\mathrm{K}}, y_{\mathrm{K}}, \theta_{\mathrm{K}}\right)}{\partial \theta_{\mathrm{K}}}}{\sum_{c_{\mathrm{K}} \in \mathbf{S}_{\mathrm{M}}} \Omega\left(c_{\mathrm{K}}, y_{\mathrm{K}}, \theta_{\mathrm{K}}\right)}-\frac{\left(\theta_{\mathrm{K}}-\theta_{\mathrm{K}-1}-\xi\right)}{\sigma_{w}^{2}}, \quad \text { (7) } 2 \leq k \leq \mathrm{K}, 1,
\end{gathered}
$$

where $\frac{\partial \Omega\left(c_{k}, y_{k}, \theta_{k}\right)}{\partial \theta_{k}}=\Omega\left(c_{k}, y_{k}, \theta_{k}\right) \frac{2}{\sigma^{2}} \operatorname{Im}\left\{y_{k} c_{k}^{*} e^{-j \theta_{k}}\right\}$. Looking for the MAP estimator implies that one must set the first derivative $\frac{\partial \ln [p(\mathbf{y}, \boldsymbol{\theta}, \boldsymbol{\xi})]}{\partial \theta_{k}}$ equal to 0 , so that:

$$
\begin{aligned}
& \theta_{1}=\theta_{2}-\xi+\sigma_{w}^{2} \frac{\sum_{c_{1} \in \mathbf{S}_{M}} \frac{\partial \Omega\left(c_{1}, y_{1}, \theta_{1}\right)}{\partial \theta_{1}}}{\sum_{c_{1} \in \mathbf{S}_{M}} \Omega\left(c_{1}, y_{1}, \theta_{1}\right)}+\sigma_{w}^{2} \frac{\partial \ln \left[p\left(\theta_{1}\right)\right]}{\partial \theta_{1}}, \\
& \left.\theta_{k}=\frac{1}{2}|| \theta_{k-1}+\frac{\sigma_{w}^{2}}{2} \frac{\sum_{c_{k} \in \mathbf{S}_{\mathrm{M}}} \frac{\partial \Omega\left(c_{k}, y_{k}, \theta_{k}\right)}{\partial \theta_{k}}}{\sum_{c_{k} \in \mathbf{S}_{\mathrm{M}}} \Omega\left(c_{k}, y_{k}, \theta_{k}\right)}\right\}+\left\{\theta_{k+1}+\frac{\sigma_{w}^{2}}{2} \frac{\sum_{c_{k} \in \mathbf{S}_{\mathbf{M}}} \frac{\partial \Omega\left(c_{k}, y_{k}, \theta_{k}\right)}{\partial \theta_{k}}}{\sum_{c_{k} \in \mathbf{S}_{\mathrm{M}}} \Omega\left(c_{k}, y_{k}, \theta_{k}\right)} \mid\right.
\end{aligned}
$$

for $2 \leq k \leq \mathrm{K}-1$,

$$
\theta_{\mathrm{K}}=\theta_{\mathrm{K}-1}+\sigma_{w}^{2} \frac{\sum_{\mathrm{c}_{\mathrm{K}} \in \mathrm{S}_{\mathrm{M}}} \frac{\partial \Omega\left(c_{\mathrm{K}}, y_{\mathrm{K}}, \theta_{\mathrm{K}}\right)}{\partial \theta_{\mathrm{K}}}}{\sum_{c_{\mathrm{K}} \in \mathrm{S}_{\mathrm{M}}} \Omega\left(c_{\mathrm{K}}, y_{\mathrm{K}}, \theta_{\mathrm{K}}\right)}+\xi .
$$

Rewrite $\frac{\sum_{c_{k} \in \mathbf{S}_{M}} \frac{\partial \Omega\left(c_{k}, y_{k}, \theta_{k}\right)}{\partial \theta_{k}}}{\sum_{c_{k} \in \mathbf{S}_{M}} \Omega\left(c_{k}, y_{k}, \theta_{k}\right)}$ as below in order to get the physical meaning of the term:

$$
\begin{aligned}
\frac{\sum_{c_{k} \in \mathbf{S}_{M}} \frac{\partial \Omega\left(c_{k}, y_{k}, \theta_{k}\right)}{\partial \theta_{k}}}{\sum_{c_{k} \in \mathbf{S}_{M}} \Omega\left(c_{k}, y_{k}, \theta_{k}\right)} & =\frac{\sum_{c_{k} \in \mathbf{S}_{M}} \exp \left\{\frac{-\left|c_{k}\right|^{2}+2 \operatorname{Re}\left\{y_{k} c_{k}^{*} e^{-j \theta_{k}}\right\}}{\sigma_{n}^{2}}\right\} \frac{2 \operatorname{Im}\left\{y_{k} c_{k}^{*} e^{-j \theta_{k}}\right\}}{\sigma_{n}^{2}}}{\sum_{c_{k} \in \mathbf{S}_{M}} \exp \left\{\frac{-\left|c_{k}\right|^{2}+2 \operatorname{Re}\left\{y_{k} c_{k}^{*} e^{-j \theta_{k}}\right\}}{\sigma_{n}^{2}}\right\}} \\
& =\frac{2}{\sigma_{n}^{2}} \sum_{c_{k} \in \mathbf{S}_{M}} \frac{p\left(y_{k} \mid c_{k}, \theta_{k}\right)}{\sum_{\tilde{c}_{k} \in \mathbf{S}_{\mathrm{M}}} p\left(y_{k} \mid \tilde{c}_{k}, \theta_{k}\right)} \operatorname{Im}\left\{y_{k} c_{k}^{*} e^{-j \theta_{k}}\right\} .
\end{aligned}
$$

One can then recognize that the factor $\operatorname{Im}\left\{y_{k} c_{k}^{*} e^{-j \theta_{k}}\right\}$ is just the classical hard decision phase detector output based on the decision $c_{k}$, while the factor $\frac{p\left(y_{k} \mid c_{k}, \theta_{k}\right)}{\sum_{\tilde{c}_{k} \in \mathbf{S}_{\mathrm{M}}} p\left(y_{k} \mid \tilde{c}_{k}, \theta_{k}\right)}$ is exactly the $a$ posteriori probability (APP) $\operatorname{Pr}\left(c_{k} \mid y_{k}, \theta_{k}\right)$ in the NDA scenario. Thus the term $\frac{\sum_{c_{k} \in \mathrm{S}_{M}} \frac{\partial \Omega\left(c_{k}, y_{k}, \theta_{k}\right)}{\partial \theta_{k}}}{\sum_{c_{k} \in \mathrm{S}_{\mathrm{M}}} \Omega\left(c_{k}, y_{k}, \theta_{k}\right)}$ can be interpreted as the soft decision phase detector output. (9) can then be written as:

$$
\left\{\begin{array}{l}
\theta_{1}=\theta_{2}-\xi+\frac{2 \sigma_{w}^{2}}{\sigma_{n}^{2}} \sum_{c_{1} \in \mathbf{S}_{\mathrm{M}}} \operatorname{Pr}\left(c_{1} \mid y_{1}, \theta_{1}\right) \operatorname{Im}\left\{y_{1} c_{1}^{*} e^{-j \theta_{1}}\right\}+\sigma_{w}^{2} \frac{\partial \ln \left[p\left(\theta_{1}\right)\right]}{\partial \theta_{1}}, \\
\theta_{k}=\frac{1}{2}\left(\theta_{k}^{(F)}+\theta_{k}^{(B)}\right) \quad \text { for } \quad 2 \leq k \leq \mathrm{K}-1, \\
\theta_{\mathrm{K}}=\theta_{\mathrm{K}-1}+\frac{2 \sigma_{w}^{2}}{\sigma_{n}^{2}} \sum_{c_{\mathrm{K}} \in \mathbf{S}_{\mathrm{M}}} \operatorname{Pr}\left(c_{\mathrm{K}} \mid y_{\mathrm{K}}, \theta_{\mathrm{K}}\right) \operatorname{Im}\left\{y_{\mathrm{K}} c_{\mathrm{K}}^{*} e^{-j \theta_{\mathrm{K}}}\right\}+\xi,
\end{array}\right.
$$




$$
\text { where }\left\{\begin{array}{l}
\theta_{k}^{(F)} \square \theta_{k-1}+\frac{\sigma_{w}^{2}}{\sigma_{n}^{2}} \sum_{c_{k} \in S_{M}} \operatorname{Pr}\left(c_{k} \mid y_{k}, \theta_{k}\right) \operatorname{Im}\left\{y_{k} c_{k}^{*} e^{-j \theta_{k}}\right\}, \\
\theta_{k}^{(B)} \square \theta_{k+1}+\frac{\sigma_{w}^{2}}{\sigma_{n}^{2}} \sum_{c_{k} \in \mathbf{S}_{M}} \operatorname{Pr}\left(c_{k} \mid y_{k}, \theta_{k}\right) \operatorname{Im}\left\{y_{k} c_{k}^{*} e^{-j \theta_{k}}\right\} .
\end{array}\right.
$$

Furthermore in (11), $\theta_{k}^{(F)}$ and $\theta_{k}^{(B)}$ can be regarded as softdecision based first-order PLL outputs which are respectively updated in the increasing (Forward) and decreasing (Backward) time directions. The physical meaning of (9) can thus be summarized as following; assuming that we do not have any $a$ priori information about the initial phase $\theta_{1}$ (i.e. $\left.\frac{\partial \ln \left[p\left(\theta_{1}\right)\right]}{\partial \theta_{1}}=0\right)$, the estimator is just estimated from $\theta_{2}$ with a backward recursion PLL; for the last position $k=\mathrm{K}$ element, the estimator is estimated from $\theta_{\mathrm{K}-1}$ with a forward PLL; and in the general case $(1<k<\mathrm{K})$, the phase estimator is estimated both from the previous and the following samples, i.e. as the average of a forward and of a backward PLL. Since in practice it is impossible to get the actual phase value $\theta_{k}$ before estimating it, we replace in (11) the true phase $\theta_{k}$ by the forward estimate $\hat{\theta}_{k-1}^{(F)}$ and the backward estimate $\hat{\theta}_{k+1}^{(B)}$ :

$$
\left\{\begin{array}{l}
\hat{\theta}_{k}^{(F)} \square \hat{\theta}_{k-1}^{(F)}+\frac{\sigma_{w}^{2}}{\sigma_{n}^{2}} \sum_{c_{k} \in \mathbf{S}_{M}} \operatorname{Pr}\left(c_{k} \mid y_{k}, \hat{\theta}_{k-1}^{(F)}\right) \operatorname{Im}\left\{y_{k} c_{k}^{*} e^{-j \hat{\theta}_{k-1}^{(F)}}\right\}, \\
\hat{\theta}_{k}^{(B)} \square \hat{\theta}_{k+1}^{(B)}+\frac{\sigma_{w}^{2}}{\sigma_{n}^{2}} \sum_{c_{k} \in \mathbf{S}_{\mathbf{M}}} \operatorname{Pr}\left(c_{k} \mid y_{k}, \hat{\theta}_{k+1}^{(B)}\right) \operatorname{Im}\left\{y_{k} c_{k}^{*} e^{-j \hat{\theta}_{k+1}^{(B)}}\right\} .
\end{array}\right.
$$

From (8) the "Forward / Backward" (F/B) estimator can thus be written as:

$$
\begin{cases}\hat{\theta}_{1}^{(F / B)}=\hat{\theta}_{2}^{(B)}-\hat{\xi}+\frac{2 \sigma_{w}^{2}}{\sigma_{n}^{2}} \sum_{c_{1} \in \mathrm{S}_{\mathrm{M}}} \operatorname{Pr}\left(c_{1} \mid y_{1}, \hat{\theta}_{2}^{(B)}\right) \operatorname{Im}\left\{y_{1} c_{1}^{*} e^{-j \hat{\theta}_{2}^{(B)}}\right\}, \\ \hat{\theta}_{k}^{(F / B)}=\frac{1}{2}\left(\hat{\theta}_{k}^{(F)}+\hat{\theta}_{k}^{(B)}\right) \quad \text { for } \quad 2 \leq k \leq \mathrm{K}-1, \\ \hat{\theta}_{\mathrm{K}}^{(F / B)}=\hat{\theta}_{\mathrm{K}-1}^{(F)}+\hat{\xi}+\frac{2 \sigma_{w}^{2}}{\sigma_{n}^{2}} \sum_{c_{\mathrm{K}} \in \mathrm{S}_{M}} \operatorname{Pr}\left(c_{\mathrm{K}} \mid y_{\mathrm{K}}, \hat{\theta}_{\mathrm{K}-1}^{(F)}\right) \operatorname{Im}\left\{y_{\mathrm{K}} c_{\mathrm{K}}^{*} e^{\left.-j \hat{\theta}_{\mathrm{K}-1}^{(F)}\right\} .}\right.\end{cases}
$$

Note that this structure is similar to that of the Kalman smoother valid for linear Gaussian problems. The name "Forward / Backward" stems from the fact that the off-line phase estimation is just the average of a classical (Forward) phase-locked loop and of a Backward phase-locked loop working in the reverse time direction and that can be initialized at the end of the forward PLL. This process can then be iterated, i.e. the estimation error at the end of the previous backward loop can be further used as the estimation error at the beginning of the next forward recursion, and several forward and backward recursions can sequentially be proceeded. We call this process in the sequel as "multiple forward / backward". Restricted by the paper size, we shall give further analysis of the proposed algorithm at the oral presentation.

\section{SimUlation AND DisCUSSION}

In a practical system, a frame header can be used and one could take advantage of it to get rid of the phase ambiguities. In our simulations, we thus assume that the phase ambiguity problem is solved. We evaluate the MSEs in the centre position of the block after $3 \mathrm{~F} / \mathrm{B}$ iterations over $10^{5}$ Monte-Carlo trials. The block length $\mathrm{K}$ for BPSK and QPSK is 60, and is 800 for the 16QAM constellation. We use the following notations in the figures of the present paragraph. "Forward (Sim)" means that the simulation MSE is measured after one (on-line) forward estimation without any backward estimation. The "Forward / Backward (Sim)" means that the MSE of the F/B estimation is measured after three (off-line) F/B iterations.

\section{A. Performance with no linear drift}

Since all the parameters are random, we compare the estimation MSE of the "Forward (Sim)" (resp. "Forward / Backward" (Sim)) with the on-line BCRB (resp. the off-line BCRB) [3],[5],[6], on Fig. 1 to Fig. 3 for different constellations.

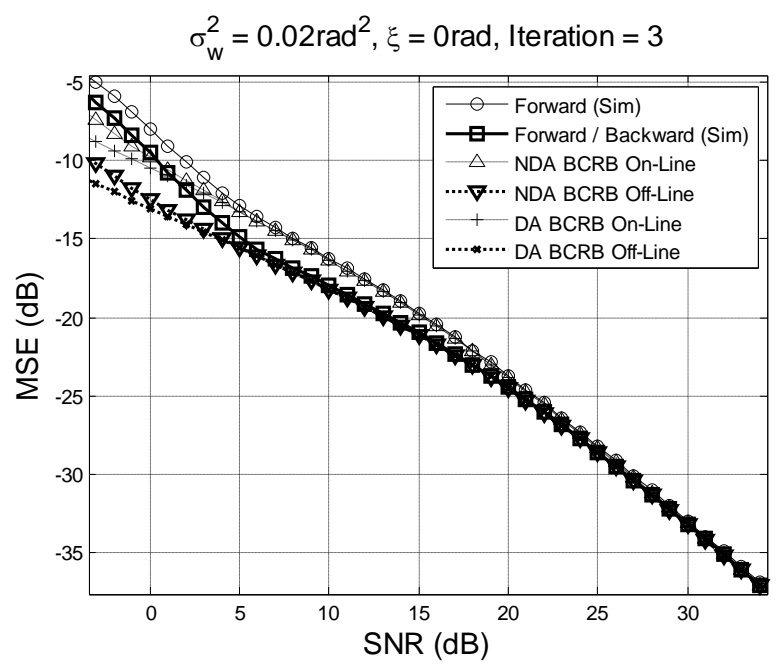

Fig. 1 BPSK MSEs and BCRBs versus SNR

At high SNR, we notice that the forward MSE and the F/B MSE curves logically merge. In this case the observations are reliable enough to only take into account the present observation $y_{k}$ in order to estimate $\theta_{k}$; this is why the off-line BCRBs (corresponding to the F/B MSE) converge to the online BCRBs (corresponding to the forward MSE), and this is also why the NDA bounds converge to the DA bounds. As the a priori distribution of $\boldsymbol{\theta}$ then has very little influence, the Bayesian problem tends to a deterministic phase estimation problem where we estimate independent observations.

In more realistic mid-range SNRs, the F/B performance is definitely superior to the forward only recursion and the maximum difference is $3 \mathrm{~dB}$. In this range of SNRs, the $a$ priori knowledge on $\boldsymbol{\theta}$ plays a very important role in the phase estimation and this is why there is a larger difference 
between the $\mathrm{F} / \mathrm{B}$ and forward recursions compared to higher and lower SNR range.

Finally, at low SNRs, because of the decision error, the MSE increases rapidly and the non-data-aided (NDA) BCRBs do not coincide anymore with the DA BCRBs. However, generally, the performance gain using a data-aided scenario is relatively low compared to the performance difference between the off-line and the on-line scenarios, and logically, when comparing with the forward recursion, there is still an appreciable gain in favor of the $\mathrm{F} / \mathrm{B}$ recursion.

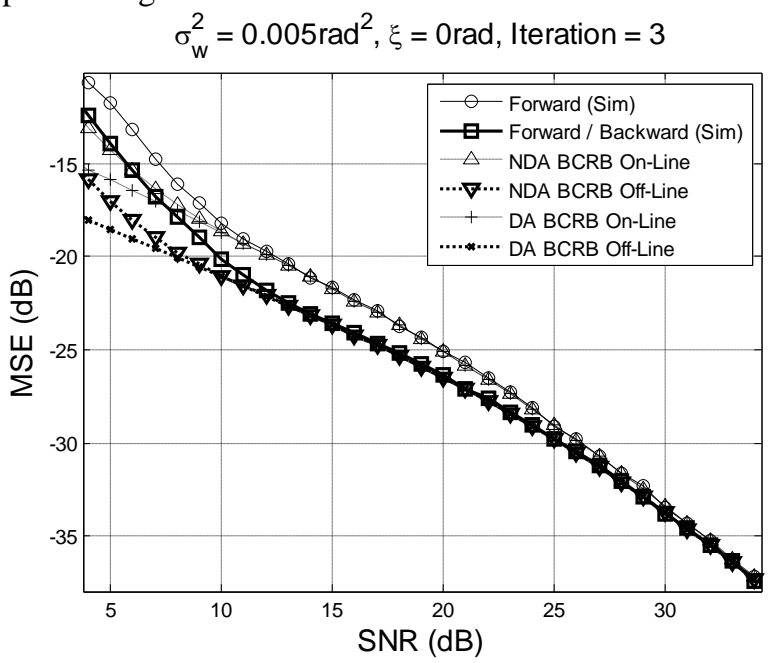

Fig. 2 QPSK MSEs and BCRBs versus SNR

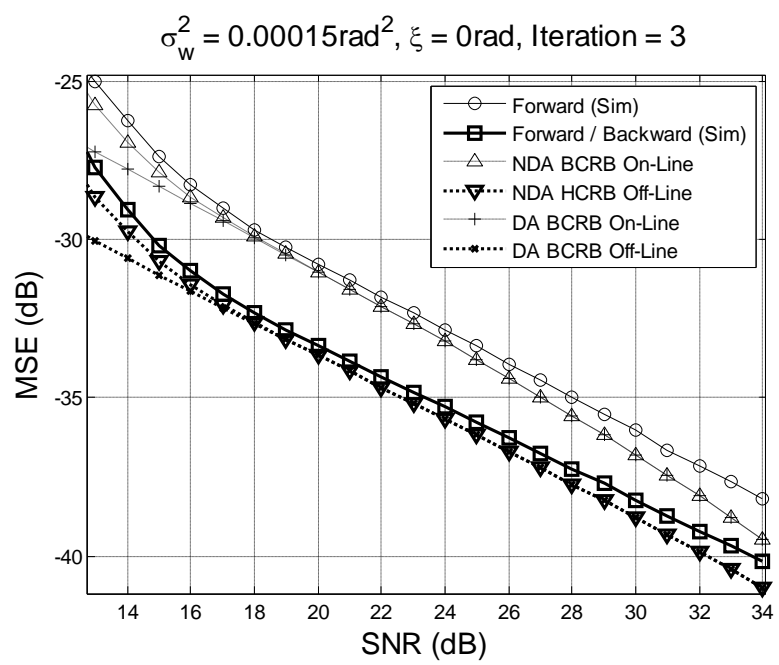

Fig. 3 16QAM MSEs and BCRBs versus SNR

\section{B. Performance with a linear drift}

Since the parameters contain both some random parameters $\theta_{k}$ and a deterministic linear drift $\xi$, we compare the MSEs to HCRBs of interest [4]-[6] for different constellations on Fig. 4 to Fig. 6.

At high SNR, the off-line HCRB coincides with the on-line HCRB, and so are the corresponding MSEs. Because in this range of SNR, the information provided by the observation is dominating over the a priori knowledge on $\boldsymbol{\theta}$, the observation $y_{k}$ is self-sufficient to estimate $\theta_{k}$ and the error on $\xi$ does not disturb the estimation performance on $\theta_{k}$.

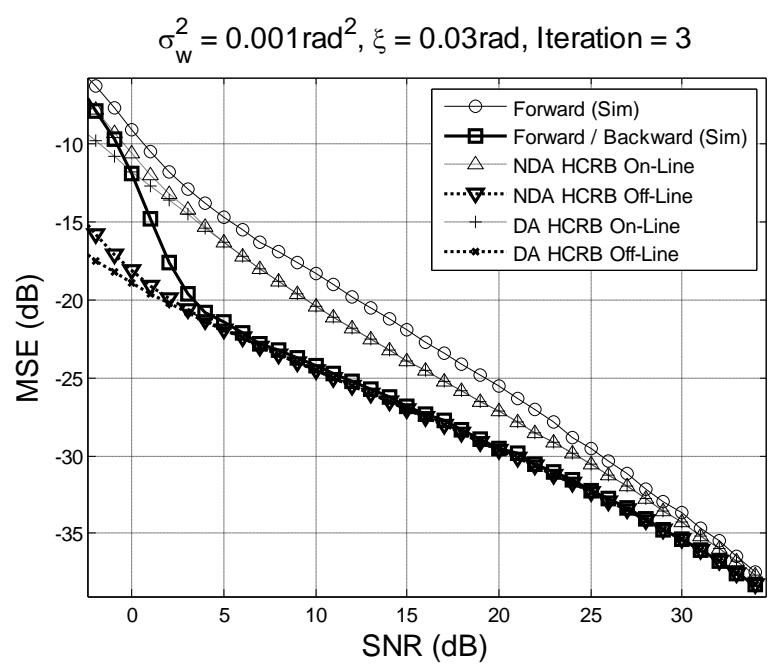

Fig. 4 BPSK MSEs and HCRBs versus SNR

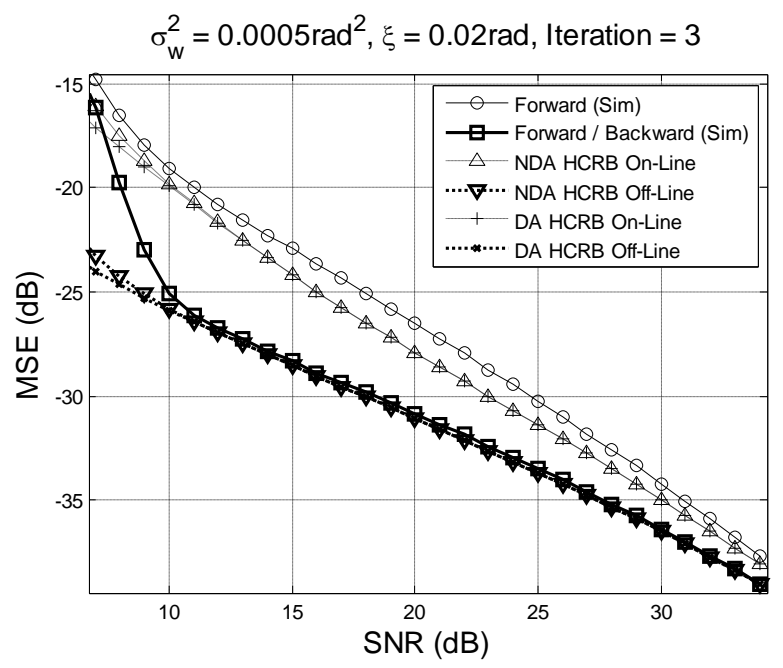

Fig. 5 QPSK MSEs and HCRBs versus SNR

At mid-range SNRs, there is not enough information provided by $y_{k}$ to estimate the phase and one can take advantage of the a priori knowledge on $\theta$ (see the difference between the on-line and the off-line BCRBs). The F/B estimation is definitely superior (up to $5 \mathrm{~dB}$ on Fig. 4) to the forward MSE not only thanks to the a priori knowledge on $\theta$; this superiority also comes from the fact that the F/B scheme remains unbiased contrarily to the forward $1^{\text {st }}$ order loop which suffers from the high linear drift as the corresponding MSE does not coincide anymore with the on-line HCRB.

At low SNRs, there is still an advantage for the $\mathrm{F} / \mathrm{B}$ recursion; however the F/B performance of Fig. 4 deteriorates rapidly, because in practice the $\mathrm{F} / \mathrm{B}$ recursion is made out of two unidirectional loops, and these loops are not able to operate 
anymore as wanted with the considered large linear drift. This phenomenon is attenuated with a smaller linear drift (see Fig. 6) or if we had replaced our simple first order PLL components by other component loops such as second order PLLs.

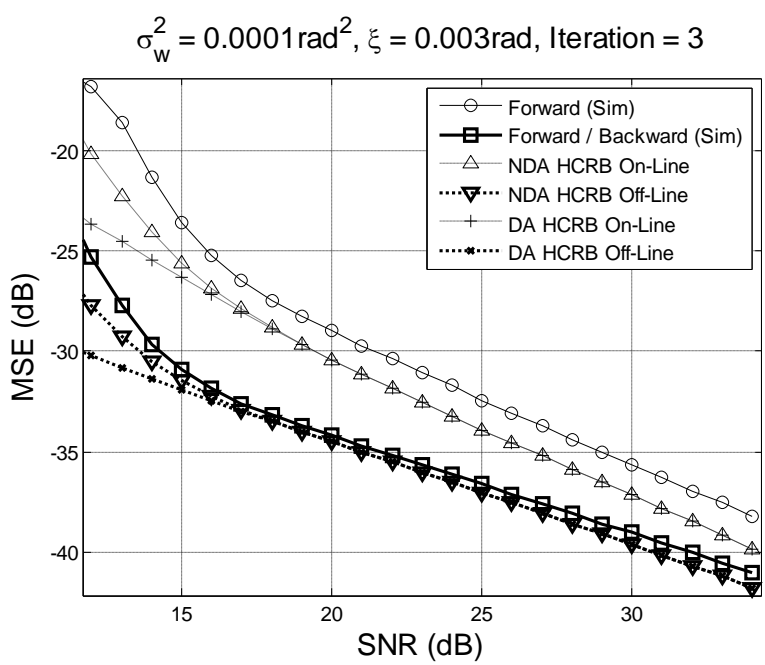

Fig. 6 16QAM MSEs and HCRBs versus SNR

\section{Algorithm complexity analysis}

The classical on-line PLL has a very low gradient-like complexity and has been employed in real systems for several decades. The complexity price for the off-line improvement is only two times that of the on-line algorithm as we combine two elementary PLLs. In addition, three forward-backward needs to be proceeded which both involves a very reasonable delay and the memorization of $\mathrm{K}$ symbols and of $2 \mathrm{~K}$ phase values.

\section{CONCLUSION}

In this paper, we presented a near-optimum smoothing phase locked loop (S-PLL) algorithm made out of two very simple first order PLLs. The performance of the S-PLL algorithm does not suffer from the poor transient behavior even with a small number of observations. The proposed scheme provides a gain of several $\mathrm{dBs}$ over a forward only on-line algorithm and its performance is near the Cramer-Rao bounds of interest. Finally it is very easy to implement and should be very useful in practice.

\section{REFERENCES}

[1] N. Noels, H. Steendam and M. Moeneclaey, "Performance Analysis of ML-Based Feedback Carrier Phase Synchronizers for Coded Signals," IEEE Transactions on Signal Processing, Vol. 55, pp. 1129-1136, March 2007.

[2] N. Noels, H. Steendam, M. Moeneclaey, "Effectiveness Study of CodeAided and Non-Code-Aided ML-Based Feedback Phase Synchronizers," Proc. IEEE Inter. Conf. on Commun. 2006, ICC'06, Istanbul, Turkey, June 11-15, 2006.

[3] S. Bay, C. Herzet, J.P. Barbot, J. M. Brossier, and B. Geller, "Analytic and Asymptotic Analysis of Bayesian Cramér-Rao Bound for Dynamical Phase Offset Estimation," IEEE Transactions on Signal Processing, vol. 56, pp. 61-70, Jan. 2008.
[4] S. Bay, B. Geller, A. Renaux, J.P. Barbot, and J.M. Brossier, "On the Hybrid Cramer-Rao bound and its application to dynamical phase estimation," IEEE Signal Processing Letters, vol. 15, pp. 453-456, 2008.

[5] J. Yang, B. Geller, and A. Wei, "Bayesian and Hybrid Cramer-Rao Bounds for QAM Dynamical Phase Estimation," in Proc. IEEE Signal Processing, ICASSP'09, Taipei, 19-24 April 2009.

[6] J. Yang, B. Geller, and A. Wei, "Approximate Expressions for CramerRao Bounds of Code Aided QAM Dynamical Phase Estimation,"in Proc. IEEE Inter. Conf. on Commun. 2009, ICC'09, Dresden, 14-18 June 2009.

[7] J. Yang and B. Geller, "Near-optimum Low-Complexity Smoothing Loops for Dynamical Phase Estimation," accepted by IEEE Trans. Signal Processing.

[8] M. Simon, W. Lindsey, "Optimum Performance of Suppressed Carrier Receivers with Costas Loop Tracking, "IEEE Trans. on Commun. vol. 25, no.2, pp. 215-227, Feb. 1977.

[9] R. E. Best, Phase-Locked Loops: Design, Simulation, and Applications, $4^{\text {th }}$ ed., McGraw-Hill, 2003..

[10] F.M. Gardner, Phaselock Techniques, $3^{\text {rd }}$ ed., Wiley-Interscience, 2005.

[11] J.M. Brossier, Signal \& Communication, Hermès, 1997.

[12] M.-L. Alberi, R.A. Casas, I. Fijalkow, and C.R. Jr. Johnson, "Looping LMS versus fast least squares algorithms: who gets there first?" $2^{\text {nd }}$ IEEE Workshop on Signal Processing Advances in Wireless Commun., SPAWC 1999.

[13] L. Zhang and A. Burr, "Iterative Carrier Phase Recovery suited for Turbo-Coded systems," IEEE Trans. on Wireless Commun., vol. 3, No. 6, pp. 2267-2276, Nov. 2004.

[14] G. Colavolpe, A. Barbieri, and G. Caire, "Algorithms for iterative decoding in the presence of strong phase noise," IEEE Journal on Selected Areas in Communications, Vol. 23, pp. 1748 - 1757, Sept. 2005.

[15] J.M. Brossier, F. Lehmann, Procédé d'estimation de la phase dans un système de communication numérique et boucle à verrouillage de phase. French patent FR20020012900, pended on the $17^{\text {th }}$ oct. 2002. International extension WO2004036753 on the $29^{\text {th }}$ april 2004. Phase Estimation Method in a Digital and Phase Locked Loop Communication System. Patent US 2006/0187894 A1. Publication date: Aug. 24, 2006.

[16] B. Geller, J.P. Barbot, J.M. Brossier, and C. Vanstraceele, Procédé d'estimation de la phase et du gain de données d'observation transmises sur un canal de transmission en modulation QAM. French patent 04P0441, pended on the $20^{\text {th }}$ sept. 2004. Method for estimating the phase of observation data transmitted over a QAM-modulated transmission channel. International extension PCT Fr 2005/02301, WCT $2006 / 032768$ on the $30^{\text {th }}$ march 2006.

[17] B. Geller, "Contribution à l'étude des systèmes de communications numériques," Chap. 3, Accreditation to Supervize Research (HDR) University of Paris, Dec. 2004.

[18] P.O. Amblard, J.M. Brossier, and E. Moisan, "Phase tracking: what do we gain from optimality? Particle filtering versus phase-locked loops," Signal Processing, vol. 83, pp. 151-167, Oct. 2003.

[19] J. A. McNeill, Jitter in ring oscillators, Ph.D. dissertation, Boston University, 1994.

[20] A. Demir, A. Mehrotra, and J. Roychowdhury, "Phase noise in oscillators: a unifying theory and numerical methods for characterization," IEEE Trans. Circuits Syst. I, vol. 47, pp. 655-674, May 2000.

[21] ETSI TR 102 376, Digital Video Broadcasting (DVB) User guidelines for the second generation system for Broadcasting, Interactive Services, News Gathering and other broadband satellite application (DVB-S2), v1.1.1, Feb, 2005.

[22] S. M. Kay, Fundamentals of statistical signal processing: estimation theory. Upper Saddle River, NJ, USA: Prentice-Hall, Inc., 1993. 